\title{
Sostenibilidad empresarial y la responsabilidad con el medio ambiente: un reto constante
}

Corporate sustainability and responsibility

to the environment: a constant challenge

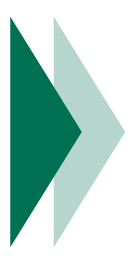

Adriana Guaqueta

Ingeniera Civil, Magister en Gerencia de Proyectos con Especialización en Gerencia de Proyectos, con amplia experiencia en el sector de la construcción e hidrocarbur

\section{Liliana Medina}

Ingeniera Civil, Magister en Proyectos, Especialista en Ingeniería Ambiental con énfasis en el área de construcción y medio ambiente, con conocimientos en aspectos normativos, elaboración de diagnósticos, análisis de información y planeación estratégica.
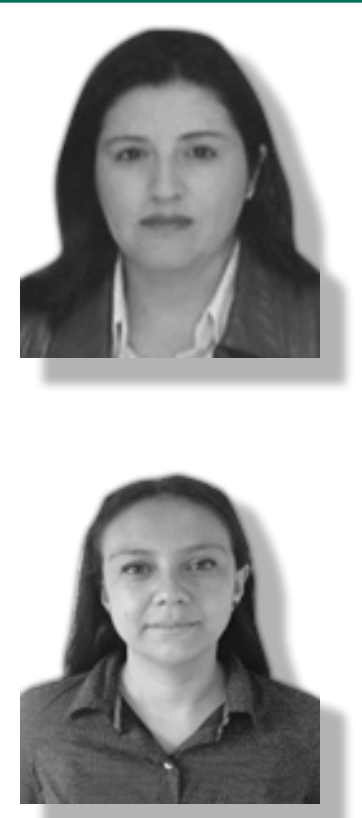
Diego Sierra

Magister en Gerencia de Proyectos, Especialista en Evaluación y Desarrollo de Proyectos, con 5 años de experiencia en el campo de proyectos, experiencia en la formulación de proyectos estatales para proyectos de Inversión, con conocimientos de la MGA.

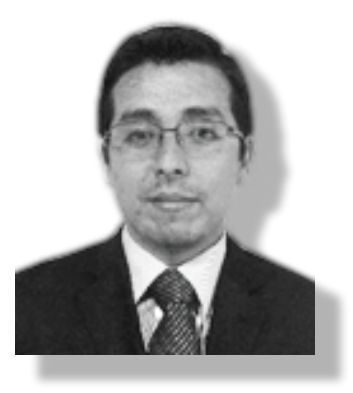

\section{RESUMEN}

Este artículo describe el significado de sostenibilidad y el auge que ha tomado, a partir del deterioro ambiental que ha venido teniendo el planeta, y cómo las empresas están emprendiendo sus proyectos y productos de manera sostenible y amigable con el medio ambiente. Adicionalmente trata el tema de la economía verde como método para que las empresas interactúen en fraternidad con el ambiente.

Palabras clave: Economía verde, sostenibilidad, sostenibilidad empresarial.

\section{ABSTRACT}

This articledescribesthemeaning of sustainability and the boom that has taken, from the environmental degradation that has been taking the planet and how companies are pursuing their projects and products environmentally friendly and sustainable manner. In addition is the theme of the green as economy so companies interact in fraternity with the environment.

Keywords: Green economy, sustainability, business sustainability.

\section{Introducción}

El planeta tierra ha tenido cambios significativos a través del tiempo, los cuáles han deteriorado los recursos naturales y estos no se están regenerando, por este motivo el mundo entero se está concientizando sobre la necesidad de un desarrollo sostenible que cubra las necesidades de la población sin comprometer el medio ambiente.

La palabra sostenibilidad ha tomado auge desde la Conferencia Mundial sobre el Medio Ambiente realizada en Estocolmo por la ONU en el año 1972, momento desde el cual el mundo empieza a tomar medidas sobre el cuidado del medio ambiente, la protección de la capa de ozono, promulgando protocolos que establezcan límites para la emisión de gases, medios prácticos para prevenir problemas ambientales, $y$, en general se empieza a crear conciencia sobre la importancia del cuidado del medio ambiente.

El desarrollo sostenible es el concepto de mayor relevancia en la actualidad que se construye a partir del desarrollo, sustentado en las dimensiones ecológicas, sociales y éticas, bases sólidas de la sociedad moderna y globalizada, dicho concepto genera una competitividad en el ámbito empresarial lo que ha generado réditos económicos para las diferentes industrias que han entrado en el concepto de generación de ingresos a través de la economía verde. El tema de la economía verde como señala Garzón Castrillón (2014): "El enfoque de la economía verde se ha orientado por dos caminos: uno es el de la concepción utilitarista centrada en productos ecológicos, bajo la premisa de que 'lo verde vende' y que lo importante es la imagen empresarial. Se plantea que en el sector empresarial los temas ambientales están cobrando mayor importancia”. 
Lo anterior nos lleva al concepto de sostenibilidad en toda su extensión como se infiere en Garzón Castrillón (2014):

No basta con el interés que las empresas puedan mostrar al medio ambiente, se requiere superar los fines utilitaristas y que además, exista una mayor conciencia del mercado consumidor para que no sólo se incline por productos ecológicos, sino que se evite consumir más de lo que en realidad se requiere, y que al mismo tiempo, se modifique el estilo de vida para contribuir con ello a un desarrollo realmente sostenible, que vaya más allá del medio ambiente y que involucre factores sociales que son medulares para lograr un desarrollo a escala humana (respeto a las minorías y conciencia social, entre otros).

El proceso sostenible de las empresas involucra el equilibrio entre el consumo de los recursos naturales y que este consumo no exceda la capacidad de regeneración del mismo.

\section{Desarrollo}

El concepto de desarrollo sostenible se debe incluir dentro de la definición de economía, que busca satisfacer las necesidades de los seres humanos a través del uso adecuado de los recursos cuando estos son escasos, este concepto ha generado crecimiento en las empresas a través de los fenómenos como la globalización generando un intercambio cultural, cambiando el pensamiento de muchas personas hacia el desarrollo sostenible apoyado en la economía verde, coadyuvando a la preservación de los recursos naturales escasos de por si, por su falta de regeneración.

Es así como la iniciativa de Economía Verde representa una potente respuesta a la petición de una acción transformadora de las empresas. El crecimiento verde consiste en abordar el cambio climático de manera decisiva a través de tecnologías e industrias verdes.

Lee Myung-Bak (2010), presidente de la República de Korea, así lo manifiesta en el artículo de la revista del Programa de Naciones Unidas para el Medio Ambiente: "Para empezar a actuar, hay que poseer conocimientos. Por lo tanto, cualquier discusión sobre el cambio climático debe incluir no solo ‘cuánto' reduciremos, sino ‘cómo' lo lograremos".

Este nuevo paradigma social y de la civilización abandona la hipótesis de negocios y estilos de vida de la era industrial, para seguir una nueva trayectoria que satisface la necesidad de crecimiento económico y la responsabilidad social corporativa, así como la integridad del medio ambiente.

Es un concepto relativamente novedoso y hace referencia a la forma de uso de recursos naturales para producir satisfacciones para la población. Sin embargo, no se ha desarrollado en todo su potencial y existen preocupaciones como crecimiento sostenible, producción, empleo y cuidado del medio ambiente y los recursos naturales. Es en resumen un nuevo paradigma en la forma de producir y en la forma de relacionarnos con el entorno.

Es así como surge la pregunta, ¿Qué es una economía verde?, en este documento se analizarán los diferentes conceptos de la economía verde, así como el crecimiento verde, de acuerdo con los puntos de vista y definiciones ofrecidas por organismos como: el Programa de las Naciones Unidas para el Medio Ambiente (PNUMA), la Organización para la Cooperación y el Desarrollo Económico (OCDE) y la Secretaria Permanente del SELA, la cual propone elementos para contribuir para una definición propia por cada región.

En términos generales podemos referirnos a que la economía verde, es aquella economía baja en carbono y eficiente en el uso de recursos naturales, además de insumos como trabajo, energía fósil y capital. Una economía verde valora e invierte en el capital natural y ofrece mejores condiciones para garantizar el crecimiento sostenible, buscando conservar y preservar el medio ambiente para garantizar las sustentabilidad de generaciones futuras (SELA, 2012).

Comparando los enfoques de las organizaciones, se encuentra que para el Sistema de Naciones Unidas, se identifica como Economía Verde, a la inversión en sectores como tecnologías eficiencia energética, energías renovables, transporte 
público, agricultura sostenible, turismo ecológico y gestión sostenible de recursos naturales, para generar nuevos sectores de producción, empleos de calidad, incrementar ingresos y mitigar en cambio climático.

Para la organización PNUMA, la Economía Verde, debe mejorar el bienestar del ser humano y la equidad social, reduciendo significativamente los riesgos ambientales. Así mismo, incorpora conceptos relacionados como Economía Positiva, orientada a eficiencia, beneficios de diferentes mercados, fomentando las energías limpias y Economía Normativa, al proponer políticas económicas indispensables para conseguir la transición a economías verdes y como mecanismo de asignación de recursos considerando elementos sociales.

En relación al enfoque de OCDE, formula una Estrategia de Crecimiento Verde, una forma de fomentar el crecimiento y el desarrollo económico y al mismo tiempo asegurar que los bienes naturales continúen proporcionando los recursos y servicios ambientales de los cuales depende el bienestar. Enfatiza el crecimiento verde como una economía de status quo, que hace surgir nuevas fuentes como productividad, oportunidades de innovación, creación de nuevos mercados, impulsar la confianza de los inversionistas, condiciones macroeconómicas más equilibradas, enmarcadas como en el concepto de Economías Positivas.

En el marco de Economías Normativas, se enfoca en políticas públicas orientadas a sustentar crecimiento verde y economía de bienestar; medición e indicadores de crecimiento verde para la toma de decisiones de las políticas económicas. Para OCDE el crecimiento verde es un complemento del Desarrollo Sostenible.

Por último para La Secretaría Permanente del SELA, una Economía Verde, necesariamente tiene que ser redistributiva y debe enfocarse en políticas que hagan influyentes el crecimiento y el desarrollo, sobre todo a los grupos más vulnerables en las áreas rurales, grupos indígenas y mujeres. La Economía Verde, es un mecanismo para llegar al Desarrollo Sostenible.
Es así como la Economía Verde, puede tener varios enfoques:

- Como sector económico.

- Como buenas prácticas para el consumo, producción sostenible, estrategias integradas y responsabilidad social.

- Conjunto de políticas para lograr objetivos de desarrollo.

- Un enfoque Integral de políticas y buenas prácticas de crecimiento y desarrollo sostenible.

Dentro de las tendencias que están marcando el desarrollo de la industria a nivel mundial está la Tendencia Verde, como la disminución en la emisión de gases con "efecto invernadero" relacionadas con la producción de energía eléctrica mediante la generación de energías renovables (tendencia oferta), y el uso más eficiente de energía generada (tendencia de demanda). - PTP 2014. Así mismo, los países están tomando conciencia sobre la responsabilidad con los recursos naturales y el aprovechamiento adecuado de los mismos, hacer negocios de forma mucho más sostenible, mejorar el bienestar humano y la equidad social y reducir de manera significativa los riesgos ambientales, como el cambio climático, y la escasez ecológica.

De otra parte cada día son más las empresas que implementan estrategias dirigidas a promover la gestión ambiental, involucrando el concepto de responsabilidad extendida desde el productor, fabricante y consumidores, implementado programas de pos consumo de residuos, uso adecuado de recursos y un alto compromiso de responsabilidad social con la filosofía de dejar un legado verde a futuras generaciones.

Sin embargo, no todos los autores desarrollan conceptos positivos referentes al término de sostenibilidad empresarial. Un grupo de pensadores analizan el concepto desde la perspectiva de los efectos negativos que puede causar la sostenibilidad empresarial.

Es así como los autores López y Méndez, en su artículo "Una crítica del concepto de desarrollo sostenible": "Critican el concepto de desarrollo sostenible por considerarlo ambiguo como fórmula para conciliar los objetivos de crecimiento económico y la proyección ambiental". Estas 
corrientes nos llevan a la dicotomía entre el crecimiento económico vs. protección ambiental (Carpenter, 1991 y 1993).

El concepto de desarrollo sostenible ha buscado reconciliar dos variables de gran importancia en el entorno mundial y que por lo general crecen con correlación negativa estas variables son; crecimiento económico y conservación ambiental. En el mundo industrializado de hoy en día durante muchos años se buscó obtener un crecimiento económico sin analizar los resultados en los que se podría incurrir con el uso desmedido de los bienes naturales no renovables, hoy en muchas partes del mundo vemos las consecuencias de un crecimiento económico que no estuvo ligado con la conservación ambiental.

Los sistemas económicos en la actualidad están basados de la producción. Las empresas deben producir cada vez más eficientemente tratando de lograr los máximos beneficios para sus accionistas. El termino de protección se "introduce" en un sistema de explotación orientado hacia la producción cuando el ritmo de esta atenta contra su condición de posibilidad, es decir, contra la posibilidad de seguir produciendo indefinidamente". Es entonces, y en el marco de una economía global, cuando tiene sentido contemplar estrategias como la de transformar la protección de la naturaleza en Ventaja competitiva (CEC, 1993).

En la perspectiva del ámbito económico global cuando cada vez los recursos naturales sean más escasos y donde el hombre no tenga la posibilidad de renovarlos esta situación se convertirá en "Sostenible" ó "Insostenible".

\section{Reflexión}

De acuerdo a todo lo aportado por los diferentes autores que hemos utilizado para construir este artículo, podemos ver que hay una diferencia marcada entre lo que desarrollan las empresas del concepto de Desarrollo Sostenible y el concepto que se le ofrece a la comunidad. La visión no es totalmente claramente para los diferentes actores que participan en la construcción del conocimiento de lo que es Desarrollo Sostenible.
La perspectiva que tiene la sociedad sobre el tema se puede indicar en los siguientes aspectos:

1. Compensar la necesidades humanas básicas.

2. Alcanzar un crecimiento económico constante

3. Optimizar la calidad del crecimiento económico.

Esta combinación de factores son lo que realmente impactan en que las personas y las empresas haciendo la diferencia entre la percepción y la realidad de lo que es el Desarrollo Sostenible, si tomamos en cuenta lo que se entiende por satisfacer las necesidad humanas básicas hace referencia a lograr evitar el hambre y la desnutrición en el mundo.

Entender que no solo hablamos de NBI, como lo son la luz, teléfono y vivienda son factores importantes para el crecimiento pero lograr la sustentabilidad alimentaria ayudaría de manera importante a evitar las brechas entre los países de primer mundo y los que están en vía de desarrollo eso hace parte de lo que es Desarrollo Sostenible.

$\mathrm{Si}$ hablamos de alcanzar el crecimiento económico constante, es lo que toda economía establecida en un país sueña para sus habitantes, generar tantos ingresos que sean capaz de reducir las brechas entre los diferentes estratos económicos, la generación de bienes y servicios de forma constante garantiza que el crecimiento poblacional no sea un factor generador de brechas en la economía, esto garantiza el crecimiento constante de la economía.

Pero dicho crecimiento debe ser con una calidad que garantice el acceso equitativo a los recursos naturales generando una redistribución equitativa de los ingresos, garantizando los beneficios sociales y la protección al ambiente y su incremento.

Todos estos aspectos que hemos mencionado solo conllevan a la generación de economías verdes donde la empresas y las naciones deben ser más comprometidas con la población que con algunas personas, porque lo que busca siempre el Desarrollo Sostenible es el acceso a la mayoría de población, de ahí que cualquier estrategia de desarrollo requiere establecer de forma la participación activa de la comunidad, ello 
implicara un esfuerzo económico importante por parte de las naciones y la busque del desarrollo en especial del sector primario que necesariamente debe estar dirigido hacia la perspectiva de la sostenibilidad.

\section{REFERENCIAS}

Burford, L. D. (2013). Project Management for Flat Organizations: Cost Effective Steps to Achieving Succesfull Results. Project Management Journal, 44(6), e4.

Chan, D., Lam, P., Chan, J., Ma, T., \& Perkin, T. (2012). A Comparative Study of the Benefits of Applying Target Cost Contracts Between South Australia and Hong Kong. Project Management Journal, 43(2), 4-20.

Commission of European Communities 1993, Biological Particles in Indoor Environments, European Collaborative Action - Indoor Air Quality and its Impact on Man, Report: 64

Emhjellen, K., Emhjellen, M., \& Osmundsen, P. (2003). Cost Estimation Overruns in the North Sea. Project Management Journal, 34(1), 23-29.

Garzón Castrillon, M. A. (2014). Revisión sobre la sostenibildad empresarial. Revista de Estudios Avanzado de Liderazgo, 1(3), 52-77.

Lawrence Pratt, L. R. (2005). El DR-CAFTA y el medio ambiente: perspectivas para el desarrollo empresarial y la sostenibilidad en Centroámerica. Revista Empresa (12), 18-28.

Meier, S. R. (2010). Causal Inferences on the Cost Overruns and Schedule Delays of LargeScale U.S. Federal Defense and Intelligence Acquisition Programs. Project Management Journal, 41(1), 28-39.

Méndez, J.A. y López, J.A. (1996). Una crítica al concepto de desarrollo sostenible. Iztapalapa. Revista de Ciencias Sociales y Humanidades 40, 123-140.
Oriol Pascual, et. al. (2011). Manual para el emprendimiento sostenible. Rotterdam: Centro Colaborador en Consumo y Producción Sostenible (CSCP).

Radi Sagbini, Z. \&. (2007). Creación de valor de las empresas colombianas durante el período 2000 - 2005. Pensamiento y Gestión, 22, 28-84.

Samset, K., \& Williams, T. (2010). Issues in FrontEnd Decision Making on Projects. Project Management Journal, 41(2), 38-49.

PHUMA, (2010). Nuestro Planeta. Revista del Programa de las Naciones Unidas, 7-8.

Secretaría Permanente del SELA. (2012). La Visión de la Economía Verde en América Latina y el Caribe, SP/Di No 1-12, 8-20 


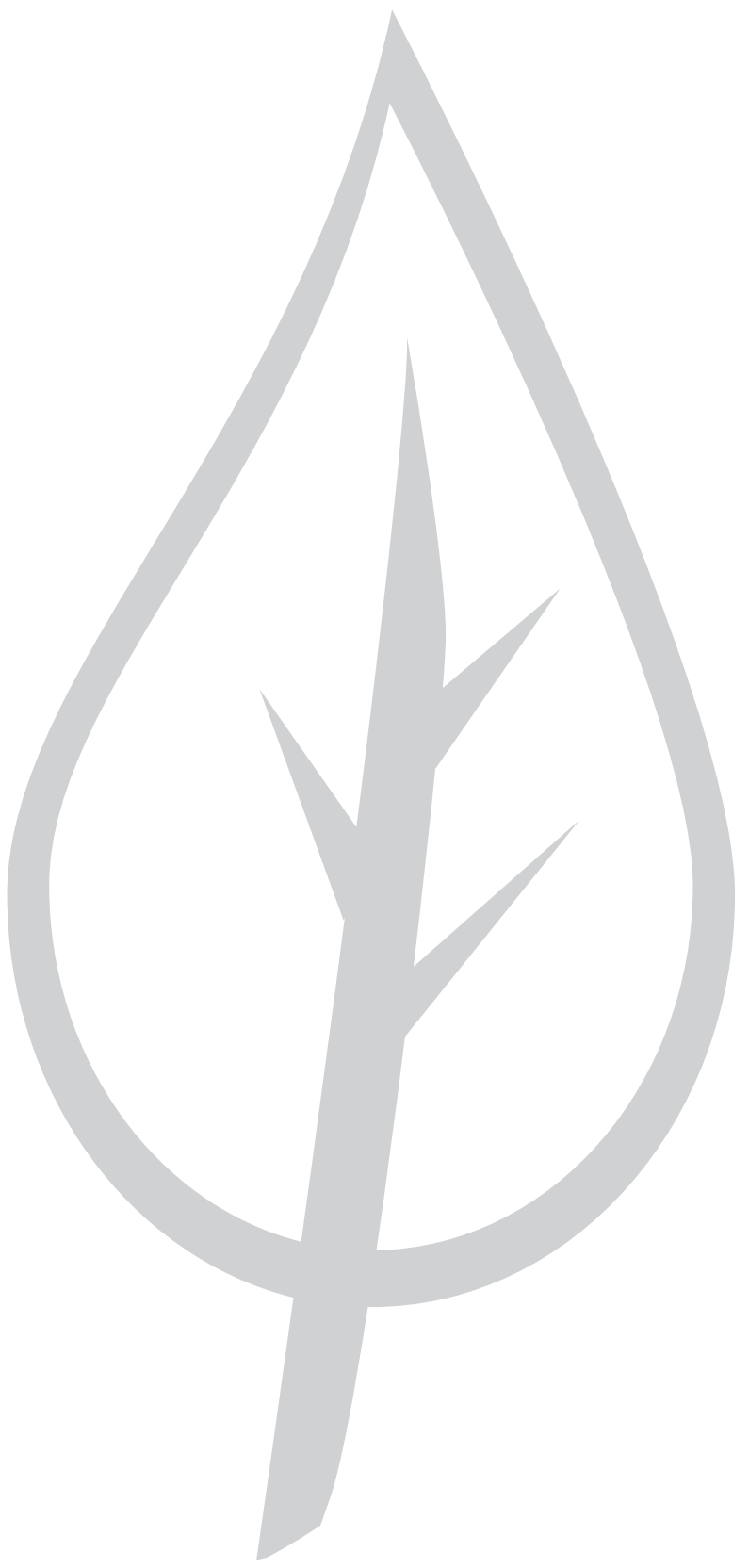

CITAS 